\title{
A new kind of locked circuit: the Quasi-Periodic Locked Loop (Q-PLL)
}

\begin{tabular}{|c|c|}
\hline Journal: & IEEE Transactions on Circuits and Systems I: Regular Papers \\
\hline Manuscript ID & TCAS-I-01682-2020 \\
\hline Manuscript Type: & Regular Paper \\
\hline $\begin{array}{r}\text { Date Submitted by the } \\
\text { Author: }\end{array}$ & $15-$ Dec-2020 \\
\hline Complete List of Authors: & $\begin{array}{l}\text { Gonzalez, Diego; Istituto per la Microelettronica e Microsistemi Consiglio } \\
\text { Nazionale delle Ricerche Unità di Bologna } \\
\text { Grassi, Lorenzo; ASPER S.r.I } \\
\text { Maurizi, Alberto; Istituto per la Microelettronica e Microsistemi Consiglio } \\
\text { Nazionale delle Ricerche Unità di Bologna, }\end{array}$ \\
\hline EDICS: & $\begin{array}{l}\text { ACS400 - Circuits and systems for biomedical applications, life science } \\
\text { and biology < ACS - Analog and Mixed Mode Circuits and Systems, } \\
\text { COMM150A5 - Multicarrier communication < COMM150 - Multichannel } \\
\text { and multicarrier systems < COMM - Circuits and Systems for } \\
\text { Communications, ACS280 - Phase locked loops < ACS - Analog and } \\
\text { Mixed Mode Circuits and Systems, ACS300 - Frequency synthesizers < } \\
\text { ACS - Analog and Mixed Mode Circuits and Systems, CTRL150A0 - } \\
\text { Control of nonlinear systems < CTRL150 - Applications of control < CTRL } \\
\text { - Control Theory topics in Circuits and Systems, NOLIN100 - } \\
\text { Analysis/modeling/simulation of nonlinear networks < NOLIN - Nonlinear } \\
\text { Circuits and Systems, NOLIN170C5 - Syncronization of chaotic systems } \\
<\text { NOLIN170 - Chaotic circuits and systems < NOLIN - Nonlinear Circuits } \\
\text { and Systems }\end{array}$ \\
\hline
\end{tabular}

\section{SCHOLARONE \\ Manuscripts}




\title{
A new kind of locked circuit: the Quasi-Periodic Locked Loop (Q-PLL)
}

\author{
Diego Luis González, Lorenzo Grassi, and Alberto Maurizi
}

\begin{abstract}
A new nonlinear circuit with frequency locking capability in the case of a generic quasi-periodic input, is presented. Due to this capability the circuit is called a Quasi-Periodic Locked Loop (Q-PLL). The locked frequency is parametrically selected from among those prescribed by the theory of resonances in dynamical systems. In particular, the locked frequency forms a three-frequency resonance with the frequencies of the quasiperiodic input. The circuit is able to lock also in case of deterministic perturbation (harmonics of the input frequencies) and stochastic perturbation (wide-band noise). The circuit is closely related to the pitch perception of complex sound in humans and, as such, can be considered a bio-inspired technology. From the point of view of applications, it may be considered as an extension of the Phase Locked Loop (PLL) with the additional ability of locking simultaneously to more than one frequency. Due to the dynamical and structural robustness of the locked states, the Q-PLL represents a tangible advance for the development of specific applications, for example, in medicine (hearing aids, and cochlear implants), in robotics (artificial senses), and in industrial and consumer electronics (improvement of speech intelligibility, pitch-based processing, etc.).
\end{abstract}

Index Terms-Quasi-periodic signals, Frequency locking, Pitch perception

\section{INTRODUCTION}

$\mathbf{P}$ HASE-Locked Loop (PLL) circuits are widely used in electronics. PLL circuits can be used as frequency multipliers or dividers, tracking generators, or clock recovery circuits. Their main technological applications derive from a capacity to recover very weak periodic signals, which would otherwise be lost in background noise, and the detection and/or synthesis of frequency and phase-controlled signals. Notwithstanding their ubiquitous presence in modern electronics, PLLs have a basic limitation in their locking capability: they can lock only to one periodic signal at a time. PLLs fail to lock when the input is perturbed by additional deterministic frequencies of competing amplitudes. However, theoretically, it is possible to have a system locked simultaneously to two or more periodic signals which are usually mutually incommensurate.

In fact, the PLL in its locked regime should be considered as a synchronized oscillator [1]-[4]. Usually the working regime is with $1 / 1$ synchronisation, in which forcing and response frequencies are the same. However, it is not unusual, depending on the kind of PLL and its operating regime, to have

Diego Luis González and Alberto Maurizi are with the Istituto di Microelettronica e Microsistemi of the Consiglio Nazionale delle Ricerche, Bologna, Italy and Dipartimento di Scienze Statistiche "Paolo Fortunati" Università di Bologna, Italy. Emails: gonzalez@bo.imm.cnr.it and alberto.maurizi@cnr.it.

Lorenzo Grassi is with ASPER S.r.1., email: lorenzo.grassi@asper-fi.it.

Correponding author: Alberto Maurizi, CNR-IMM, via Gobetti 101, 40129 Bologna, Italy; email: alberto.maurizi@cnr.it. locked states corresponding to other synchronised conditions such as, for example, $1 / \mathrm{n}$, where the loop is locked to a sub-harmonic of the forcing signal. The locked responses are indeed resonances of a system with two frequencies (twofrequency resonances).

Dynamical systems theory provides a framework for systems with $n$-frequency resonances. It has been demonstrated [5] that locking in the case of 3-frequency resonances is possible, i.e., the response synchronises with two independent forcing frequencies.

Moreover, it has also been shown that three-frequency resonances are related to a relevant aspect of neural dynamics, namely the pitch perception of complex sounds [6], [7]. This aspect is relevant for applications because it opens up the possibility of technological developments in various fields including medicine, artificial senses, and robotics.

Our main objective here is to develop, on the basis of a biologically oriented paradigm, a loop circuit able to lock simultaneously to two independent frequencies. Such a circuit would extend the capabilities of the well-known phase-locked loop (PLL) and overcome the structural stability limitation of previously studied dynamical systems (coupled nonlinear oscillators, coupled phase-locked loops and coupled circle maps [5], [8], [9]). This new non-linear circuit is termed a QuasiPeriodic Locked Loop (Q-PLL, patent pending [10], [11]) due to its main property, that is, its ability to stably lock on a quasi-periodic input given by two independent frequencies. The circuit is interesting for applications envisaging real time responses of the auditory system, and also shows promise for other electronic applications representing a significant step forward with respect to standard PLL circuits.

First, the properties of three-frequency resonances from a dynamical systems point of view will be recalled and their implications for pitch perception outlined. The structural stability problems of known implementations will be discussed. Then, the circuit architecture will be presented and the strategy of the implementation for obtaining robust three-frequency resonant responses will be explained. After this, the response of the Q-PLL circuit in parameter space will be studied and its deterministic (signal perturbations) and structural (parameter perturbations) stability properties analysed. Finally, several implementations and possible future developments and applications will be discussed.

\section{THREE-FREQUENCY RESONANCES AND PITCH PERCEPTION}

Synchronization was discovered by Christiaan Huygens in 1665 [12]. He found that the pendulums of two clocks, fixed 
on the same mounting, after some time swung synchronously. Moreover, Huygens found that the phases of clock oscillations showed an asymptotic tendency towards a certain fixed value of the phase difference. Because of this tendency, synchronization is also known as phase locking.

Synchronization is a well-known universal phenomenon occurring widely in nature, from heartbeats to the movement of celestial bodies [13], [14]. The most common case, well described by the Huygens clocks, is when the forcing and response frequencies are equal. This is called 1/1 synchronization and corresponds, for example, to the case of orbital and rotational synchronised frequencies characterizing the movement of our moon and responsible of the fact that we see always the same face.

Synchronization can appear in dynamical systems with more than one external periodic forcing. Consider the general case of a nonlinear system forced by $n$ independent external forces. For $n=1$, a periodic response corresponds to a synchronized, or locked, state. As said above, this resonance can differ from simple 1/1 synchronization. In fact, when the quotient between the proper frequency $f_{0}$ of the unperturbed system and the frequency $f_{1}$ of the external forcing, approach a rational number, i.e., $f_{0} / f_{1} \simeq p / q$ with $p, q \in \mathbb{N}$, a solution characterized by a sub-harmonic of the forcing frequency, i.e., $f_{1} / q$ can arise. When the response locks exactly to the $q$ subharmonic, then $f_{0} / f_{1}=p / q$ and thus $p f_{0}-q f_{1}=0$. For $p=q=1$, it reduces to $1 / 1$ synchronization which implies $f_{0}=f_{1}$. This last relationship is a special case of the more general case represented by

$$
\sum_{k=0}^{N-1} m_{k} f_{k}=0
$$

where $m_{k}$ are integers. A system which satisfies Equation (1) non-trivially, i.e., $m_{k} \neq 0$ for all values of $k$, is said to be $N$-resonant (the frequencies form an $N$-resonance).

Analog phase-locked loop circuits represent an electronic realization of a 2-resonant system showing responses locked to the forcing frequency, i.e., the case with $N=2$ and $\left|m_{k}\right|=1$ in Equation 1.

The next case in increasing dimensionality $(N=3)$ corresponds to quasi-periodic forcing, i.e., a system forced with two independent external frequencies $f_{1}$ and $f_{2}$. Quasiperiodically forced systems exhibit a rich variety of dynamical behaviour [15]. These range from periodic behaviour, to 2 - and 3 -frequency quasi-periodic responses, to strange non-chaotic, and chaotic attractors. The Ruelle-Takens-Newhouse theorem establishes that quasi-periodic attractors with 3 incommensurate frequencies are on the border of chaos. These quasiperiodic attractors imply that Equation (1) with $N=3$ has only the trivial solution ( $m_{k}=0$ for all $k$ values). The theorem implies that in the immediate neighborhood of these responses, chaotic attractors must be present. As a consequence, quasiperiodic attractors with three-frequencies would be unstable in parameter space.

However, when Equation (1) has non-trivial solutions for $N=3$, the three frequencies satisfy a resonant condition, i.e., are not independent and, therefore, the Ruelle-Takens-

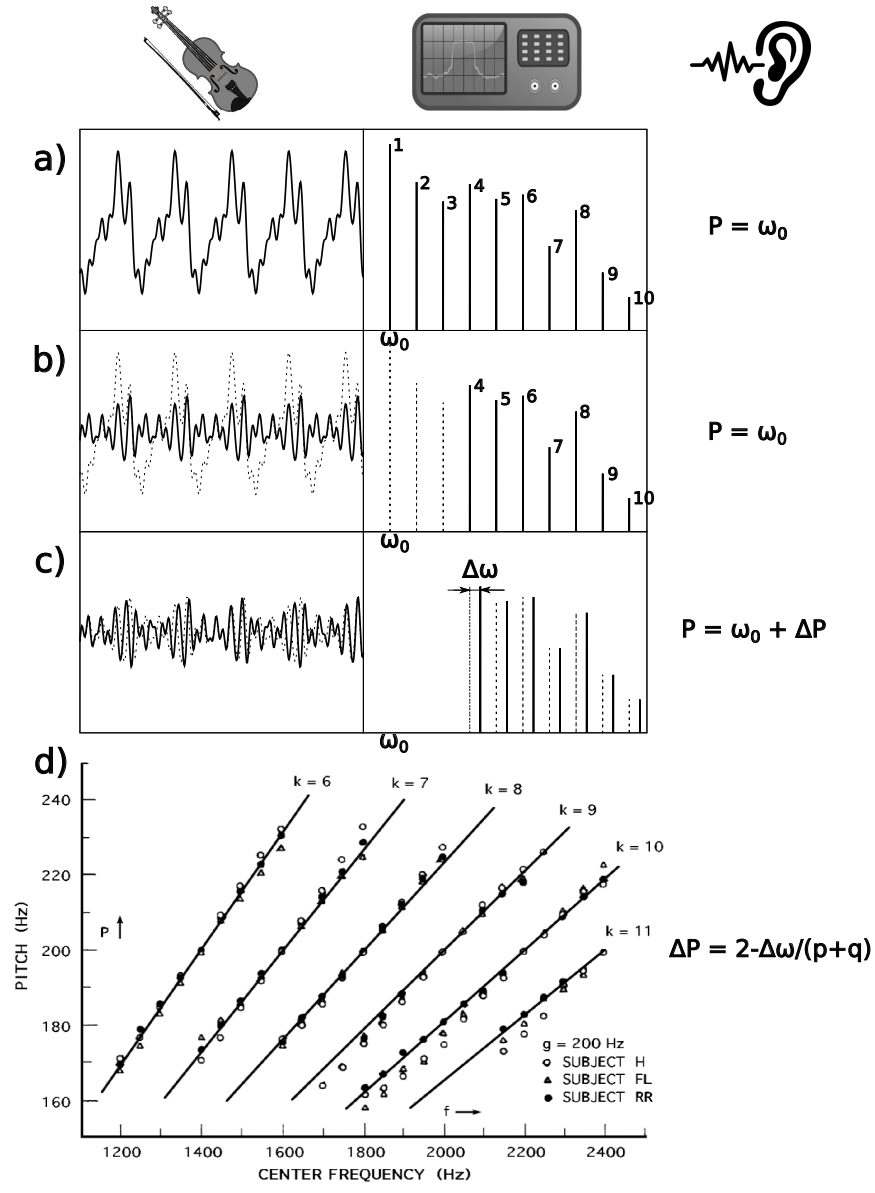

Fig. 1. Schematic representation of the relationship between tones and pitch for different stimuli. Musical sounds are complex tones consisting of a lowest frequency, called fundamental ( $\omega_{0}$ here) and integer multiples of it, called overtones or harmonics. Panel a) reports the complex tone time series and power spectrum of the first 10 harmonics of a violin whose pitch concides with the fundamental. In panel b) the fundamental and the first few higher components of the spectrum are removed. The pitch does not change with respect to a): it remains that of the fundamental (missing fundamental). Panel c) displays the effect of uniformly shifting the harmonics of panel b) by a quantity $\Delta \omega$. Although the difference combination tones remain unchanged, the pitch shifts by a quantity $\Delta P$. Panel d) reports the relationship between the frequency shift and the perceived pitch according to [6] superimposed on data from the pitch shift experiment in [16].

Newhouse theorem does not apply. Thus, the system is expected to have resonant responses with stability regions of non-zero size in parameter space. This has already been shown to hold in several nonlinear quasi-periodically forced systems [8].

Although in the study of nonlinear dynamical systems the characterization of deterministic chaos has attracted a great deal of attention, more regular dynamics are found to be important for modelling many real processes. In particular, the study of 3-frequency resonances was found to be particularly important for modelling the auditory perception of complex sounds [6], [7].

The pitch of a complex sound is a psychoacoustic quantity, i.e., a subjective placement of the sound along an inner template that goes from bass to high tones, as in a musical scale. If a correspondence between pitch and the frequency spectrum of the stimulus is sought, for the majority of musical 
sounds, i.e., those composed of a fundamental and a series of harmonics, the task is relatively easy: the pitch can be well described by the fundamental component of the sound (see Figure 1a). However in some cases, the pitch is not trivially connected to the lowest frequency of the stimulus. In fact, even when a few of the lowest components of a musical sound are removed, the perceived pitch still corresponds to that of the (removed) fundamental (see Figure 1b). This phenomenon is called missing fundamental or residue. In order to explain this behaviour, a static nonlinear theory was formulated by Helmholtz [17]: the difference tone (one of the possible combination tones), produced within the ear, plays the role of the missing fundamental: the difference between two adjacent partials equals the fundamental component. As an example, in Figure $1 \mathrm{~b}, f_{5}-f_{4} \equiv 5 f_{0}-4 f_{0}=f_{0}$. Nevertheless, pitch shift experiments, consisting of the observation that the pitch shifts when all the frequency components are shifted by the same amount (see Figure 1c where it is shown that the pitch is also shifted by the amount $\Delta P$ ) [16], demonstrated the fallacy of this view (Figure 1c). This fact was intepreted historically as a failure of the nonlinear approach [18]-[20]. However, more recently, a nonlinear dynamical theory [6], [7] (as opposed to the static view of Helmholtz) was successful in explaining the key feature of pitch shift (Figure 1d). This new theory is based on the concept of dynamical resonances. The main results of the theory and its application to pitch perception modelling are briefly outlined here below (see [6], [7] for a more detailed description).

When a generic dynamical system characterized by an intrinsic frequency $f_{0}$ is forced by two external independent frequencies $f_{1}$ and $f_{2}$, a web of three-frequency resonances is generated in its parameter space. The general procedure for finding the possible resonances starts with the continued fraction development of the quotient of the external frequencies, $r=f_{1} / f_{2}$ (see, e.g., [21]):

$$
r=\left[a_{0} ; a_{1}, \ldots, a_{n}, \ldots\right] .
$$

The development is finite if the quotient is rational and infinite otherwise. Successive truncations up to a given finite order of the development give the so-called approximants of the ratio,

$$
r_{n}=\frac{p_{n}}{q_{n}}=\left[a_{0} ; a_{1}, \ldots, a_{n}\right] .
$$

For any approximant a main three-frequency resonance exists which is given by:

$$
f_{0_{n}}=\frac{f_{1}+f_{2}}{p_{n}+q_{n}}
$$

The hierarchy of the frequencies $f_{0_{n}}$ characterizing the web of three-frequency resonances is described by a generalized Farey sum operation. The usual Farey sum operation on rational numbers, $(p / q) \oplus(r / s)=(p+r) /(q+s)$, describes the hierarchy of the web of synchronized responses in periodically forced oscillators (two-frequency resonances). Such hierarchical organization is known as the Devil's staircase.

Analogously, for three-frequency resonances, there is a hierarchical organization given by the generalized Farey sum operation [8] defined as follows: for a given convergent

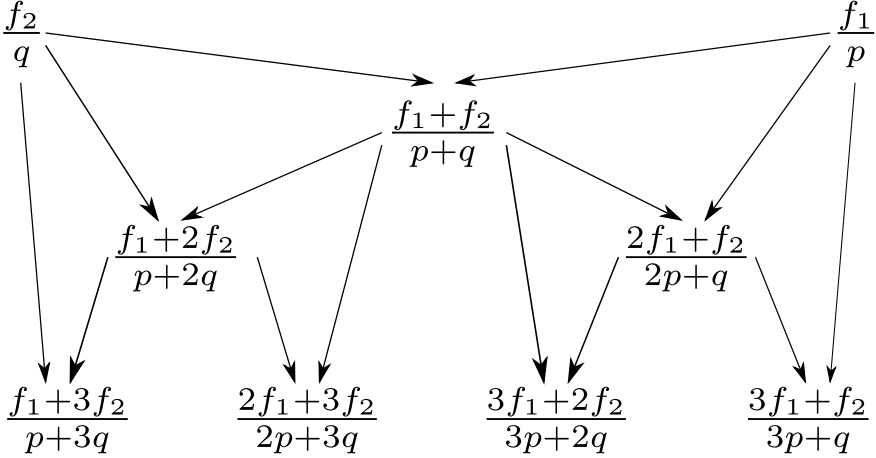

Fig. 2. Sketch of the first few levels of the generalized Farey sum hierarchy.

$r_{n}=p_{n} / q_{n} \equiv\left(p_{n}, q_{n}\right)$, define an interval of frequencies $\left[f_{1} / p_{n} ; f_{2} / q_{n}\right]$. Then, the first step of the generalized Farey sum is defined as

$$
\frac{f_{1}}{p_{n}} \oplus \frac{f_{2}}{q_{n}}=\frac{f_{1}+f_{2}}{p_{n}+q_{n}} .
$$

The operation can be iterated between adjacents, taking into account that two frequencies $f_{x}$ and $f_{y}$ are considered as adjacents if $s f_{x}-r f_{y}=q f_{1}-p f_{2}$. At variance with the usual Farey sum, the generalized Farey sum acts on quotients between (mixed) real and integer numbers. In this way, a hierarchical organization of resonances (similar to that corresponding to periodically forced oscillators) is formed in generic quasi-periodically forced systems [22]. The first steps of this hierarchy, based on the generalized Farey sum, are sketched in Figure 2. The fundamental fact here, is that the pitch of complex sounds, including the phenomenon of missing fundamental, can be described in terms of three-frequency resonances. In particular, the pitch elicited by a quasi-periodic stimulus corresponds to a main three-frequency resonance. In the simplest case of a two frequencies stimulus, the pitch is represented by a three-frequency resonance obtained as the generalized Farey sum between the subharmonics $f_{1} / p$ and $f_{2} / q$ where $p / q$ is an approximant of the stimulus frequency ratio. Such a description is in excellent agreement with experimental psycho-acoustical data explaining, with high precision, the phenomenon of pitch shift (see Figure 1c and d).

Having a robust dynamical model for the prediction of the pitch of complex sounds may be very useful in some applications. However, from the practical point of view, a major drawback appears: the stability intervals corresponding to the main three-frequency resonances appear to be very narrow in all the dynamical systems studied so far: 1) nonlinear coupled oscillators with piecewise analytical solutions, 2) coupled PLLs [9], 3) quasi-periodically forced circle-maps [5], [8]. This is due to the fact that the web of resonances existing in the narrow interval determined by $f_{1} / p$ and $f_{2} / q$ is very complex (in principle, infinite resonances, which correspond to the successive levels of the generalized Farey sum operation, can coexist). This is similar to the case of the infinite steps of the Devil's staircase in periodically forced systems.

In order to tackle this stability problem, a system which can stably produce these main three-frequency resonances over wide intervals of parameter space is required. This implies that 


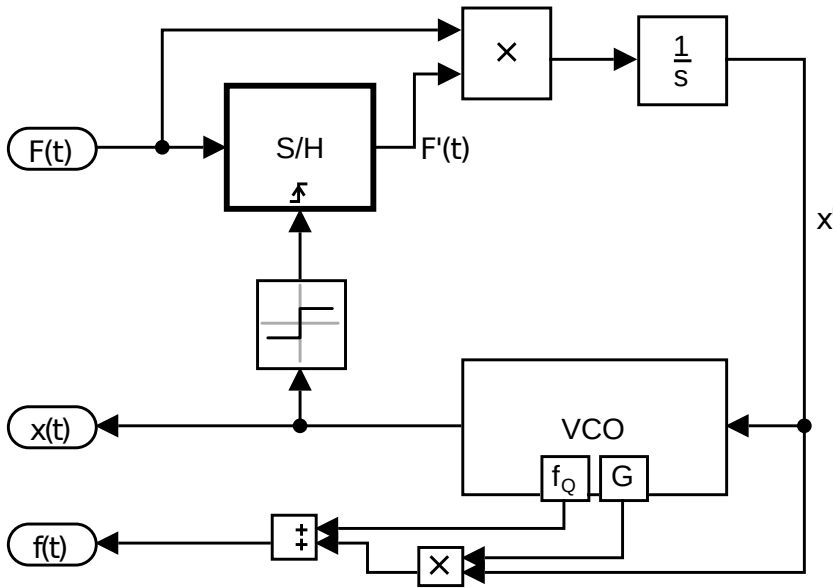

Fig. 3. Implementation of Q-PLL in Simulink®. The input signal $F(t)$ (see Equation (6) for symbols) enters the $\mathrm{S} / \mathrm{H}$ module whose output $F^{\prime}(t)$ is multiplied by $F(t)$ itself and then integrated over time (low-pass filtered) to give $x^{\prime}(t)$ which, in turn, determines the VCO frequency. The output of the system $x(t)$ is the locked signal which stems from the VCO. The instantaneous frequency $f(t)$ of the output signal is also given, obtained using the same formula implemented in the VCO. The sign function between the VCO output and the S-H trigger input, is used to transform a sine wave to an impulse in order to trigger the $\mathrm{S} / \mathrm{H}$.

some kind of dynamic control must be implemented in order to enhance the existence intervals of the main resonances. The following Section describes an electronic circuit, based on a sampling locked loop control displaying very large stability intervals for the main three-frequency resonances, and which can lock not only on periodic but also on quasi-periodic inputs.

\section{CIRCUIT DESCRIPTION}

The Q-PLL circuit robustly implements three-frequency resonator dynamics. The main idea underlying the circuit design is to use a sub-Nyquist approach taking into account that the desired three-frequency resonances are below the Nyquist frequency of the forcing. Within this framework, a feedback system is developed which locks stably to one of the possible three-frequency resonances.

In Figure 3 a possible realization of the circuit in terms of a Simulink ${ }^{\circledR}$ model is shown. It also represents well the general functional scheme of the system. The quasi-periodic input signal $F(t)$ is sent to a triggered sampler (Sample and Hold, $\mathrm{S} / \mathrm{H}$ ). The sampling frequency of the $\mathrm{S} / \mathrm{H}$ is determined by the output frequency of a Voltage Controlled Oscillator (VCO). The sampled signal $F^{\prime}(t)$ is then multiplied $(\times)$ by the non-sampled one and, subsequently sent to a time integrator $(1 / \mathrm{s})$. Then, the integrated signal $x^{\prime}(t)$ is sent to the VCO to determine the VCO frequency as $f_{\mathrm{VCO}}=G x^{\prime}(t)+f_{Q}$, where $G$ and $f_{Q}$ are the sensitivity and quiescent frequency of the VCO, respectively. The output of the VCO, once transformed into a square wave, is sent to the trigger port of the $\mathrm{S} / \mathrm{H}$. The response of the system is the output signal of the VCO $x(t)$ with instantaneous frequency $f(t)$.

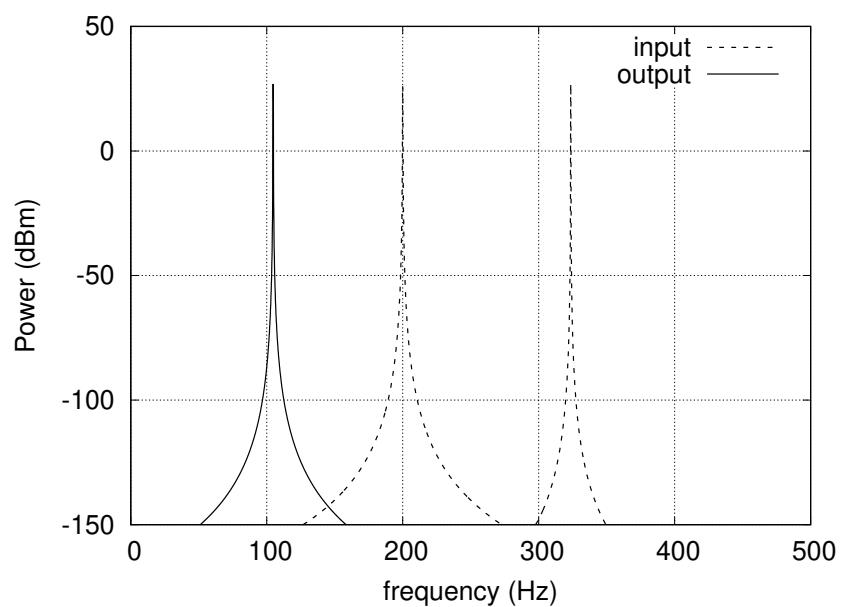

Fig. 4. Example of locking for quasi-periodic input: $f_{1}=200 \mathrm{~Hz}$, $f_{1} / f_{2}=\phi$, the golden ratio, and for VCO parameters: $f_{Q}=100 \mathrm{~Hz}$ and $G=2000 \mathrm{~V} / \mathrm{Hz}$ (NB: the result is independent of the initial phase $\varphi_{0}$ ). The only surviving frequency in the output signal is the "residue" frequency corresponding to $f_{0} \simeq 104.72 \mathrm{~Hz}$, according to three-frequency resonance theory as outlined in Section II.

The equation that describes the dynamics of the Q-PLL shown in Figure 3, can be written as:

$$
x(t)=A_{0} \cos \left(2 \pi\left[f_{Q}+G \int_{0}^{t} F(\tau) F^{\prime}(\tau) \mathrm{d} \tau\right] t+\varphi_{0}\right)
$$

where $\varphi_{0}$ is the initial phase of the VCO, $F(t)$ is the input (forcing) and $F^{\prime}(t)$, the sampled signal, is formally expressed by $F^{\prime}(t)=F\left(t_{i}\right)$ for $t_{i} \leq t<t_{i+1}$ with $t_{i}=\left\{t \mid \mathrm{d}_{t} \mathcal{H}(x(t))>0\right\}$ where $\mathcal{H}$ is the Heaviside function and $\mathrm{d}_{t}$ is the distributional derivative. The sampled signal function $F^{\prime}(t)$ makes this equation, analytically speaking, rather intractable. Features of the model will be studied using numerical simulations.

Figure 4 reports, as an example, the power spectrum of the Q-PLL output (continuous line) along with that of the input signal (dashed line) for an input $f_{1}=200 \mathrm{~Hz}$ and $f_{2}=\phi f_{1}$ where $\phi=(1+\sqrt{5}) / 2$ is the golden ratio. This irrational number ensures that the frequency ratio is as far as possible from a rational ratio (quasi-periodic forcing).

In the general case, the sampling frequency $f_{s} \equiv f_{\mathrm{VCO}}$, including its initial value $f_{Q}$, is below the Nyquist frequency of the input.

It is well known from sampling theory (see, e.g., [23]), that sampling a sinusoidal signal of frequency $f_{1}$ with a sampling frequency $f_{s}<2 f_{1}$ produces a spectrum with aliasing components. The sequence $f_{a_{k}}$ of aliases (on the positive frequency axis) is based on the quantity $\Delta_{1} \equiv\left|f_{1}-\hat{k}_{1} f_{s}\right|\left(\hat{k}_{1}\right.$ being the nearest integer to $f_{1} / f_{s}$.):

$$
f_{a_{k}}=\left\{\Delta_{1}, \mp \Delta_{1}+k f_{s} ; k \in \mathbb{N}\right\} .
$$

As a direct consequence, for $k=\hat{k}_{1}$, it results that either $\Delta_{1}+\hat{k}_{1} f_{s}=f_{1}$ or $-\Delta_{1}+\hat{k}_{1} f_{s}=f_{1}$, in other words, the resulting signal contains the input frequency.

If the signal to be sampled (input signal) is a superposition of two sinusoids of frequencies $f_{1}$ and $f_{2}$ satisfying the condition $f_{s}<2 \min \left\{f_{1}, f_{2}\right\}$, the power spectrum of the 


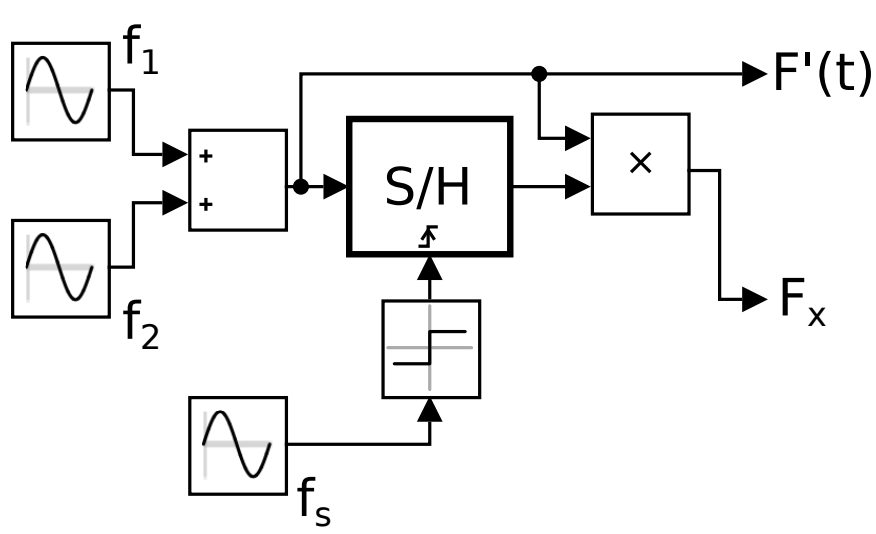

Fig. 5. Scheme of the sampling system implemented in Q-PLL. Two sine signals of frequencies $f_{1}$ and $f_{2}$ are added and constitute the input signal. Sampling is triggered in $\mathrm{S} / \mathrm{H}$ when a sine wave of independent frequency $f_{s}$ crosses the zero from negative values. The signals to be analysed for the Q-PLL locking mechanism are the sampling output $F^{\prime}(t)$ and the signal resulting from the product of the input and the sampled signal $F_{\times}$.

sampled signal, for linearity, is the superposition of two power spectra containing aliasing components based on $\Delta_{1}$ and $\Delta_{2}=\left|f_{2}-\hat{k}_{2} f_{s}\right|$.

Thus, according to the sub-Nyquist sampling properties just recalled, the sampled signal contains components at frequencies $f_{1}$ and $f_{2}$ coinciding with the input components. Taking the product $(\times)$ of the two signals (input and sampled) produces a signal $F_{\times}$with a non-null zero-frequency component $\mathcal{F}(0)$ of the power spectrum. This Direct Current (DC) term is the sum of the contribution resulting from the zero-frequency components, $\mathcal{F}_{1}(0)$ and $\mathcal{F}_{2}(0)$, of the two input signals.

Furthermore, along with the DC component, the two lowest alias frequencies $\Delta_{1}$ and $\Delta_{2}$ constitute the low frequency part of the spectrum which survives the subsequent low-pass filtering produced by the integrator. The analysis of these quantities allows a deeper insight into the locking mechanism as shown below.

Consider the open submodel, i.e., without feedback, shown in Figure 5, where the triggering signal (input to the $\mathrm{S} / \mathrm{H}$ module) is replaced by a controlled frequency $f_{s}$. The Fourier transform of the product signal $F_{\times}$is computed and the zerofrequency component $\mathcal{F}(0)$ is considered. Calculation of this quantity is performed for different values of the sampling signal frequency $f_{s}$ over a range covering the convergence frequency interval, while its phase $\varphi_{s}$ is varied over the full range $[0: 2 \pi]$. Results are reported in Figures $6-8$.

To gain an idea of how the locking mechanism acts, it is important to observe that a necessary condition for the output of the Q-PLL circuit in Figure 3 to lock to a fixed value, is that the input to the VCO be constant. Therefore, the input signal to the integrator must vanish. A necessary condition for this is that the DC component of $F_{\times}$vanishes. Figure 6 displays the DC component, $\mathcal{F}(0)$, over a wide range of $f_{s}$ that cover the first three approximants of the frequency input ratio. The shaded areas enclose the intervals defined by $\left[f 1 / p_{k}, f 2 / q_{k}\right]$, for $\left(p_{k}, q_{k}\right)=(1,2),(2,3),(3,5)$, with $p_{k} / q_{k}$ being the $k$ -

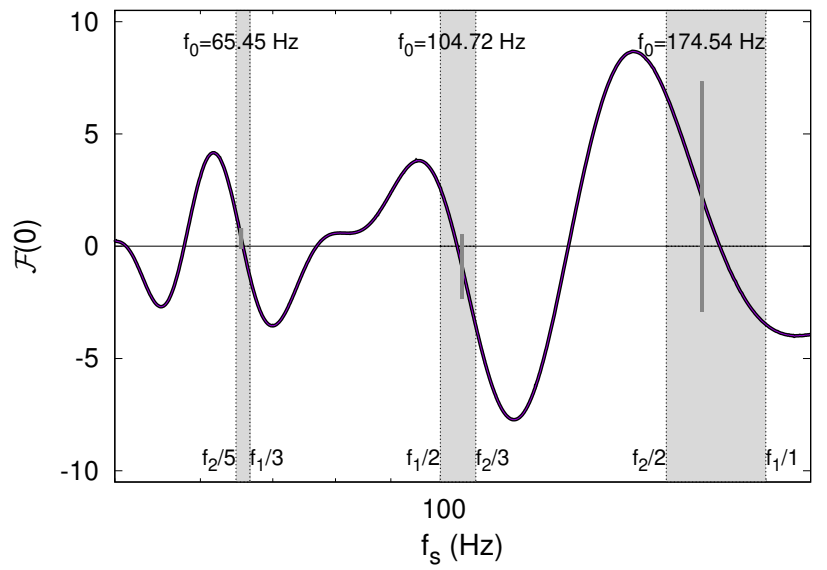

Fig. 6. DC component $\mathcal{F}(0)$ (in arbitrary units) and convergence intervals for the first three approximants. It can be observed that the value of $\mathcal{F}(0)$ alternates its sign moving between adjacent convergents. The shaded regions represent the area bounded by $f_{1} / p$ and $f_{2} / q$. It is worth noting that the order of $f_{1} / p$ and $f_{2} / q$ alternates between adjacent convergents. Frequency of the first mediant $f_{0}=\frac{f_{1}+f_{2}}{p+q}$ is reported for each convergence interval.

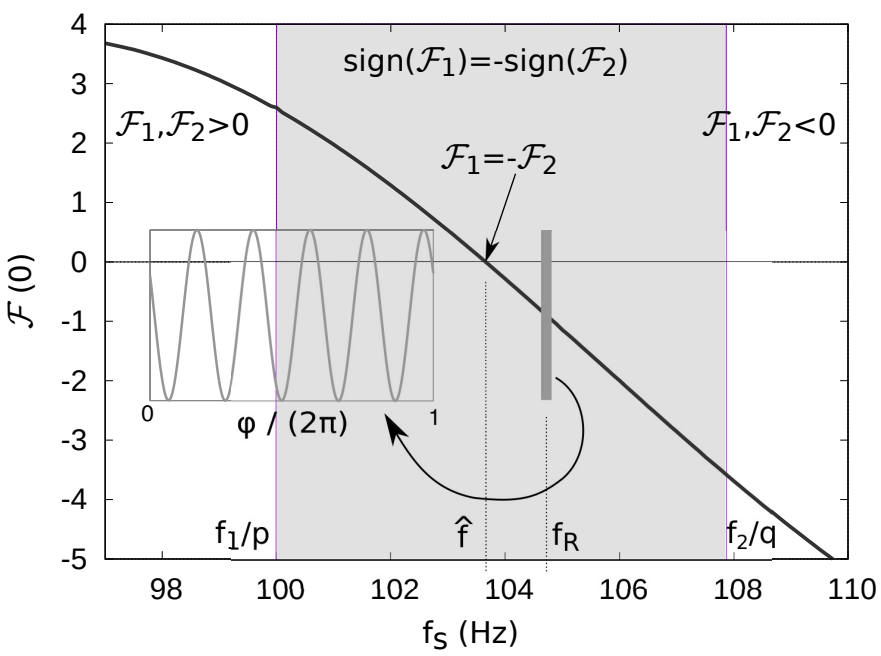

Fig. 7. DC component $\mathcal{F}(0)$ (in arbitrary units) of the product of input and sampled $\left(F_{\times}\right)$signals over a range of frequencies around $f_{0}$ for convergent $\left(p_{k}, q_{k}\right)=(2,3)$. Input frequencies are $f_{1}=200 \mathrm{~Hz}$ and $f_{2}=\phi f_{1}$. Shaded area shows the range between $f_{1} / p$ and $f_{2} / q$ ). Vertical line at $f_{s}=f_{0}$ is the variation of $\mathcal{F}$ due to the change in phase $\varphi_{s}$ which occurs only at that frequency. The dependence on this second variable is shown in the figure insert which displays, with the same units, the variations in $\mathcal{F}$ with $\varphi_{s}$. It can be observed that for the rest of the plot, changes in $\mathcal{F}$ depend only upon $f_{s}$. Labels indicate the relationship between $\mathcal{F}_{1}$ and $\mathcal{F}_{2}$ for different ranges of $f_{s}$.

th convergent of $f_{1} / f_{2}$, and $k=1,2,3$. The vertical lines represent the variations in $\mathcal{F}(0)$, caused by sampling signal phase change, which the system produces at exactly $f_{s}=f_{0 k}$ (and only for those values). To closely analyse this behaviour, Figure 7 displays a zoom of Figure 6 around the convergence region of the second approximant $\left(p_{k}, q_{k}\right)=(2,3)$. Consider the values of the two components of $\mathcal{F}(0)$, namely $\mathcal{F}_{1}$ and $\mathcal{F}_{2}$ : they are in some relationship with $f_{s}$. Outside the shaded region in Figure 7, for $f_{s}<f_{1} / p$ they are both positive while they are both negative for $f_{s}>f_{2} / q$. Inside the shaded region, 


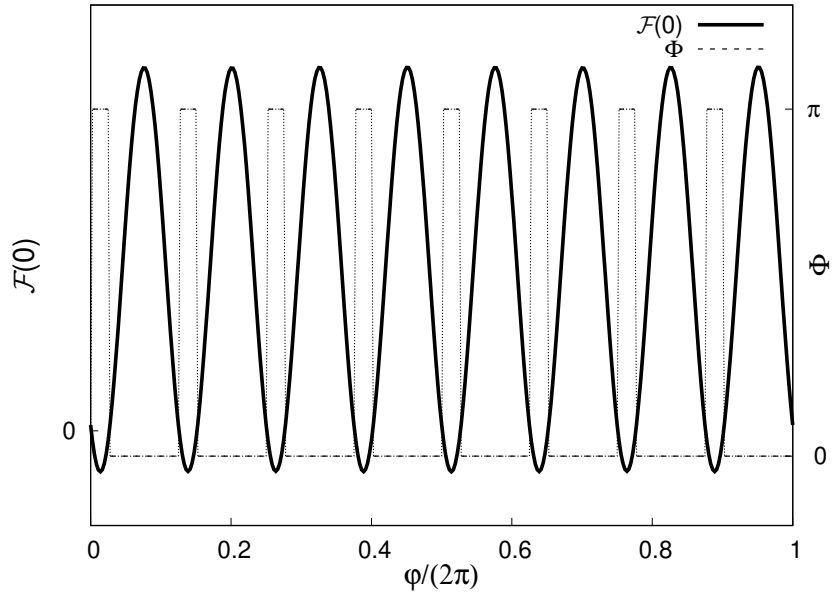

Fig. 8. Phase response for $f_{s}=f_{0}$ for convergent $(p, q)=(3,5)$ in case of input frequencies $f_{1}=200 \mathrm{~Hz}$ and $f_{2}=\phi f_{1}$. Thick line is the DC component (in arbitrary units) while the thin dashed line is the phase $\Phi$ of the response. It is important to observe that the number of cycles is exactly $p+q$ (compare to the insert in Figure 7). Moreover, the output phase only plays the role of the sign function, as it jumps from 0 to $\pi$ when the DC component changes its sign.

their sign is opposite and, for a given $f_{s}=\hat{f}$, they equal each other in magnitude making $\mathcal{F}(0)$ vanish. However, Figure 7 shows that $\hat{f}$ does not correspond to the main resonance and, therefore, the locking mechanism must rely on some additional features of the system.

In fact, $\mathcal{F}(0)$ is, in general, a function of both the frequency $f_{s}$ and the phase $\varphi_{s}$ of the sampling signal, and Figure 7 should be viewed as a projection along the $\varphi_{s}$ axis. However, it can be observed that over the range represented in Figure 7, except for $f_{s}=f_{0}, \mathcal{F}(0)$ does not depend on $\varphi_{s}$. Nevertheless, a strong dependence appears for $f_{s}=f_{0}$ as shown by the thick vertical line in Figure 7. It is known from Section II that in this case $\Delta_{1}=\Delta_{2}$ and the two alias frequency sequences coincide. The plot inserted in Figure 7 represents the section of $\mathcal{F}(0)$ along the $\varphi_{s}$ axis at fixed $f_{s}=f_{0}$ and displays explicitly this dependence. It is important to observe that the variations of $\mathcal{F}(0)$ due to $\varphi_{s}$ are such that $\mathcal{F}(0)$ crosses the zero value, thus providing a mechanism of convergence towards the main resonance frequency $f_{0}$.

It is remarkable that this very special phase dependence happens exactly at $f_{s}=f_{0}$, which underlines the strong connection between three-frequency resonances and the properties of the signal $F_{\times}$and, in turn, with the sub-Nyquist-sampled signal $F^{\prime}$. Another remarkable feature is that the periodicity of $\mathcal{F}(0)$ as a function of $\varphi_{s}$ is exactly $p+q$. The insert in Figure 7 corresponds to the convergent $\left(p_{k}, q_{k}\right)=(2,3)$ and the function presents 5 cycles. As another example of this feature, Figure 8 shows the case corresponding to the convergent $\left(p_{k}, q_{k}\right)=(3,5)$ for which the periodicity of the phase response results to be 8 .

Consider now the closed circuit (Figure 3 ). At the beginning of the locking cycle, if $f_{Q}<\min \left(\hat{f}_{k}, f_{0_{k}}\right)$, the resulting DC is positive and forces the system to increase the sampling frequency $f_{s}$ (in the opposite case, if $f_{Q}>\max \left(\hat{f}_{k}, f_{0_{k}}\right) f_{s}$ decreases). When $f_{s}$ crosses $f_{0_{k}}$, a phase-locking mechanism is activated and the system adjusts the phase of the sampling signal to find one of the zeroes of the DC component (Figures 7 and 8). This corresponds to a relative phase which cancels the two low frequency components $\left(\Delta_{1}=\Delta_{2}\right)$ of the aliases, ensuring a zero input to the integrator. When far from the resonance, the two low frequency components $\left(\Delta_{1} \neq \Delta_{2}\right)$ provide a perturbation which leads the system to cross $f_{s}=f_{0_{k}}$ and then to switch to the phase-locking mechanism described above. The parameter acting as an amplifier of the perturbation is the VCO sensitivity $G$ which, if too small does not allow the system to move far enough from the $\mathrm{DC}=0$ solution and cross the resonance value $f_{0}$. On the other hand, if $G$ is too large the circuit becomes unstable.

\section{EXPERIMENTAL SETUP AND RESULTS}

The circuit features were studied by means of numerical experiments. The implementation of the circuit in terms of Simulink $®$ blocks shown in Figure 3 is used here.

As already shown in the example reported in Figure 4, the system is able to lock to a quasi-periodic input composed of two incommensurate sinusoidal components whose ratio, notably, is the "most irrational" number, i.e., the golden ratio $\phi$. The ability to lock to different convergents of the $f_{1} / f_{2}$ ratio over the entire range of the parameter $f_{Q}$, is investigated here below.

Before proceeding further, some considerations on the dependence of the system on $G$ are in order. $G$ provides the rate of change of the VCO frequency as a function of the integrated signal. Roughly speaking, it must depend on the expected frequency response which, in turn, depends on $f_{Q}$. This can be observed through examining the intervals of the DC component around the locking frequencies in Figure 6. As a rule of thumb, the smaller the interval extent, the smaller $G$ must be in order to avoid jumps outside the given convergence basin and to maximize the existence regions of the main resonances. On this basis, appropriate values for $G \equiv \tilde{G}_{\left(p_{k}, q_{k}\right)}$ are selected for each of the $k$-th approximant $\left(p_{k}, q_{k}\right)$.

Thus, by means of a trial-and-error procedure several values of $\tilde{G}_{\left(p_{k}, q_{k}\right)}$ were selected: $G_{(1,2)}=8000 \mathrm{~Hz} / \mathrm{V}, G_{(2,3)}=$ $2000 \mathrm{~Hz} / \mathrm{V}, G_{(3,5)}=1000 \mathrm{~Hz} / \mathrm{V}, G_{(5,8)}=500 \mathrm{~Hz} / \mathrm{V}$, $G_{(8,13)}=250 \mathrm{~Hz} / \mathrm{V}$. Small variations of $G$ around the optimal values defined above have no significant effect and, therefore, this parameter is not investigated further.

\section{A. Q-PLL features for quasi-periodic input}

Figure 9 reports the response frequency $f_{R}$ of the Q-PLL as a function of $f_{Q}$. It can be observed that $f_{R}$ locks exactly to $f_{0_{k}}$, the main three-frequency resonance corresponding to the $k$-th convergent. The extent of the intervals over which the solutions are stable are very large, much larger than the $\left[f 1 / p_{k}, f 2 / q_{k}\right]$ intervals reported as continuous thin lines in Figure 9 (see also the shaded regions in Figure 6). It is worth noting that all other possible solutions corresponding to generalized mediants in the Devil's staircase-like portrait (see Figure 2) expected to exist between $f_{1} / p_{k}$ and $f_{2} / q_{k}$, are suppressed. This is at variance with what has been found for some systems previously investigated: coupled phase-locked 
1

2

3

4

5

6

7

8

9

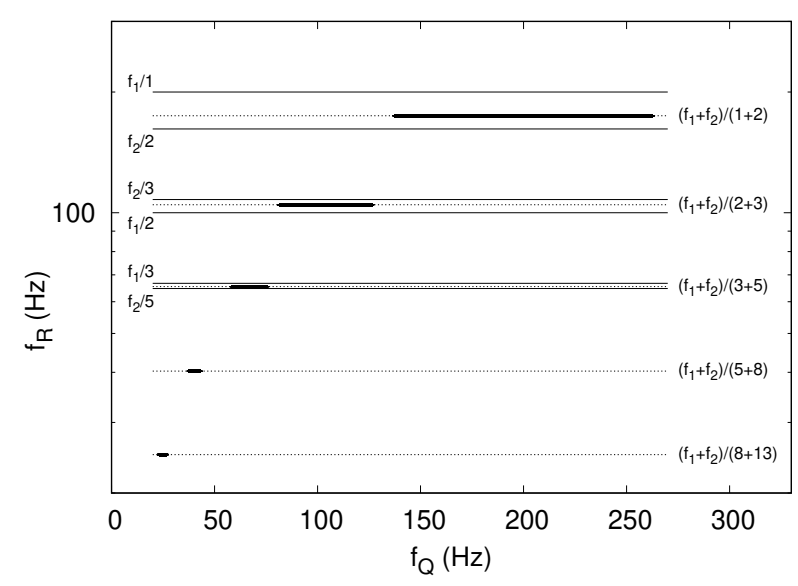

Fig. 9. Response diagram as a function of $f_{Q}$ for optimal values of $\tilde{G}_{(p, q)}$. Input frequencies are: $f_{1}=200 \mathrm{~Hz}$ and $f_{2}=f_{1} \phi$. The frequency value corresponding to the first mediant $\left(f_{1}+f_{2}\right) /(p+q)$ (dotted lines) of the bounding values $f_{1} / p$ and $f_{2} / q$ for the Devil's staircase (continuous thin lines) are shown (the latter not shown from $(p, q) \geq(5,8)$ ). Solutions (thick lines) converge to the first mediant. The convergence intervals width increases from high to low $p+q$. Regions of $f_{Q}$ for which $f_{R}$ is not given, are regions of instability of the solution.

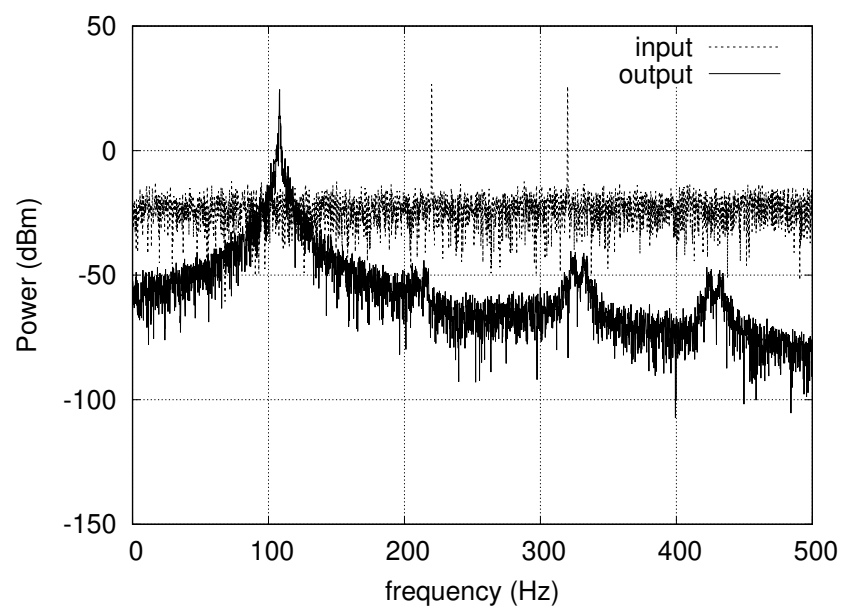

Fig. 10. Example of locking in response to a signal perturbed by noise. Input is as in Figure 4 with additive white Gaussian noise of $-45 \mathrm{~dB}$ relative to the peaks.

loops, coupled nonlinear oscillators with piece-wise analytic solution and a quasi-periodically forced circle map [5], [8], [9]. Within the convergence intervals the attractors are stable and, therefore, evolve to asymptotic orbits independently of the initial conditions, mainly the initial phase of the VCO.

The highest reported convergent is $\left(p_{6}, q_{6}\right)=(8,13)$. Convergence intervals corresponding to higher convergents are too short to be represented fairly on the same scale. At the frontier of the reported stable solutions there are small regions in which the system is unstable where the responses may depend on the initial conditions. In these regions, however, other stable solutions may also occasionally appear (not reported here) which correspond to generalized mediants of the neighbouring (parent) solutions (see Figure 2).

Experiments to study the stability of the main resonances

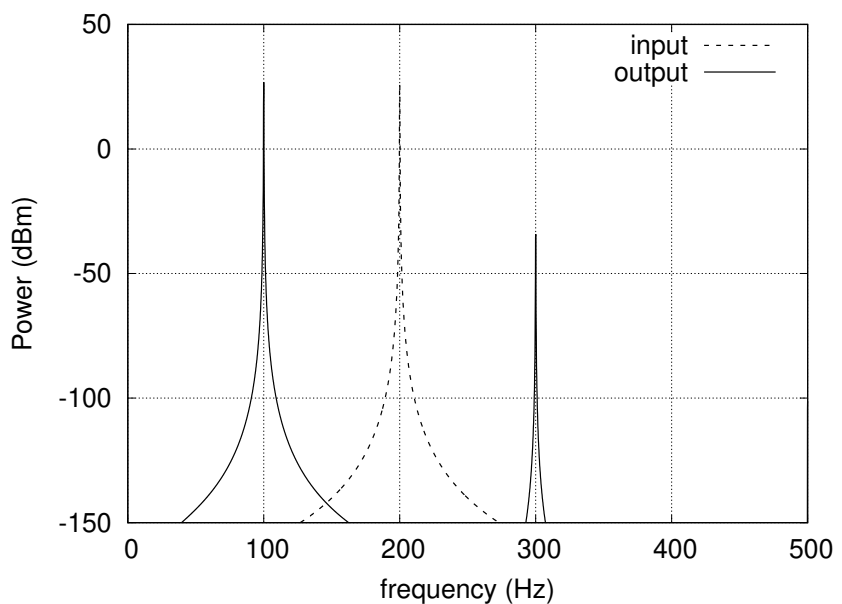

Fig. 11. Example of locking for one frequency input only (PLL-like behaviour) for $f_{1}=200 \mathrm{~Hz}$ and $f_{Q}=90 \mathrm{~Hz}$. Output is the input frequency $f_{1}$ divided by $n=2$. Q-PLL also produces harmonics of the response frequency of much lower amplitude.

facing various perturbations of the input signals were also performed. First, the signal of Figure 4 was perturbed by an additive white Gaussian noise with an intensity of about $-30 \mathrm{dBm}$ relative to the input $(0 \mathrm{dBm})$. For the present system implementation and reported parameters, the system response remains stable for noise figures below or equal to this level. This can be appreciated as a peak corresponding to the expected resonance at $104.72 \mathrm{~Hz}$ in Figure 10. A thorough investigation about the stability to noise perturbation is beyond the scope of this paper but would undoubtedly be interesting for the design of electronics applications.

\section{B. PLL-like behaviour}

If the Q-PLL is forced with a signal containing a frequency $f_{1}=200 \mathrm{~Hz}$ only, selecting $f_{Q}$ from a suitable range around $f_{Q}=200 \mathrm{~Hz}$ the system locks at $f_{R}=200 \mathrm{~Hz}$ as would a normal PLL.

If now the quiescent frequency is set, for example, to $f_{Q}=$ $90 \mathrm{~Hz}$, the system locks to $f_{R}=100 \mathrm{~Hz}$ (Figure 11). This frequency is the first sub-harmonic of the forcing frequency, $f_{R}=f_{1} / 2$. In general, the system is able to lock to any subharmonic $f_{R}=f_{1} / n$ depending on the value of $f_{Q}$. Figure 12 shows the response diagram as a function of $f_{Q}$. Solutions are very stable and do not depend on the VCO sensitivity $G$ (in Figure $12, G=1000 \mathrm{~Hz} / \mathrm{V}$ is used). In this situation the QPLL behaves, in the limit, as a PLL with frequency-divider capability such as, for example, those in the sampling class of PLLs.

\section{Q-PLL as a pitch detector}

As the focus of this work is on the ability of the circuit to model the pitch of complex sounds, its ability to lock in the case of the sum of two sine waves with harmonic frequency ratio $f_{1} / f_{2}=2 / 3$ is analysed. In musical terms, this particular ratio corresponds to a perfect fifth interval. Such a ratio is used as a paradigmatic example being considered one of the most 


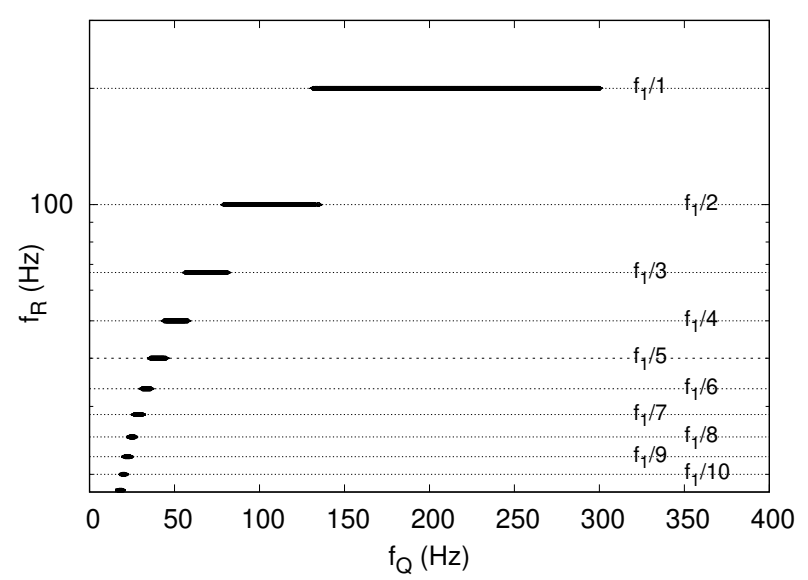

Fig. 12. Response diagram for one input frequency only (PLL-like behaviour) as a function of $f_{Q}$, for $f_{1}=200 \mathrm{~Hz}$. Depending on the VCO quiescent frequency various sub-harmonics of $f_{1}$ can be selected.

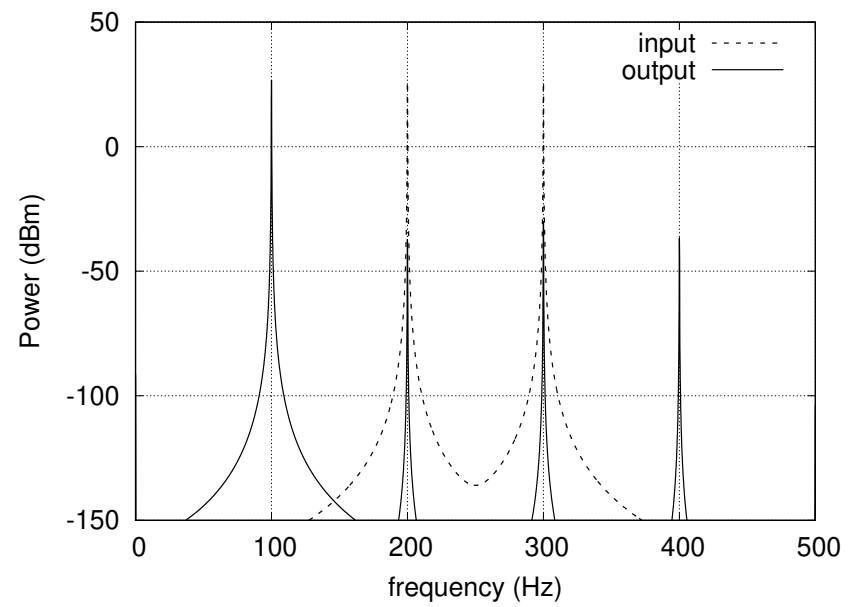

Fig. 13. Example of locking for harmonic input: $f_{1}=200 \mathrm{~Hz}, f_{2}=300 \mathrm{~Hz}$ and $f_{Q}=90 \mathrm{~Hz}$. Harmonics of the response frequency are also produced with lower amplitude.

consonant musical intervals. It should be borne in mind that this interval represents a stimulus with a missing fundamental at $f_{1} / 2 \equiv f_{2} / 3$.

Figure 13 displays the input and response frequencies for the perfect fifth case, $f_{1}=200 \mathrm{~Hz}$ and $f_{2}=300 \mathrm{~Hz}$ with $f_{Q}=$ $90 \mathrm{~Hz}$. The system locks stably to the missing fundamental value $f_{R}=100 \mathrm{~Hz}$.

Next, the more complex case corresponding to a pitchshift is analysed. Figure 14, reports the result for a nonharmonic signal obtained by applying a pitch shift operator to the harmonic signal used in the perfect fifth example. A small shift $\Delta f=21 \mathrm{~Hz}$ was selected to keep the ratio $2 / 3$ as the first approximant of the input frequency ratio. In fact, the continued fractions development of the ratio $221 / 321$ is:

$$
[1,2,4,1,3,5]
$$

and the corresponding convergents: $1,3 / 2,13 / 9, \ldots$

Figure 14 shows the power spectrum density of the input and output signals. The system locks exactly (within the numerical

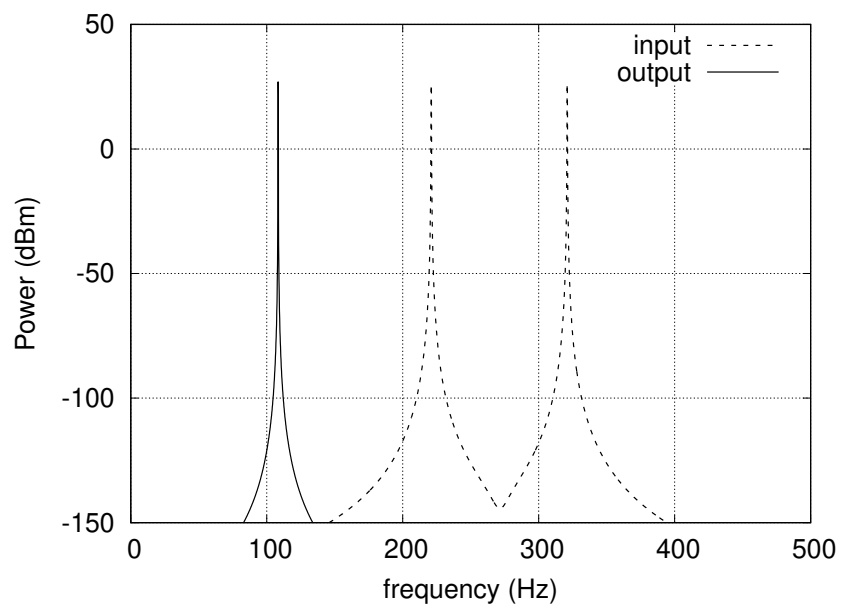

Fig. 14. Example of locking for inharmonic input ("pitch shift" of $\Delta f=$ $21 \mathrm{~Hz}$ with respect to Figure 13): $f_{1}=221 \mathrm{~Hz}, f_{2}=321 \mathrm{~Hz}$ and $f_{Q}=$ $90 \mathrm{~Hz}$. The only surviving frequency in the output signal is the "residue" frequency corresponding to $f_{0}=\left(f_{1} / 2+f_{2} / 3\right)=108.4 \mathrm{~Hz}$, according to Equation (4)

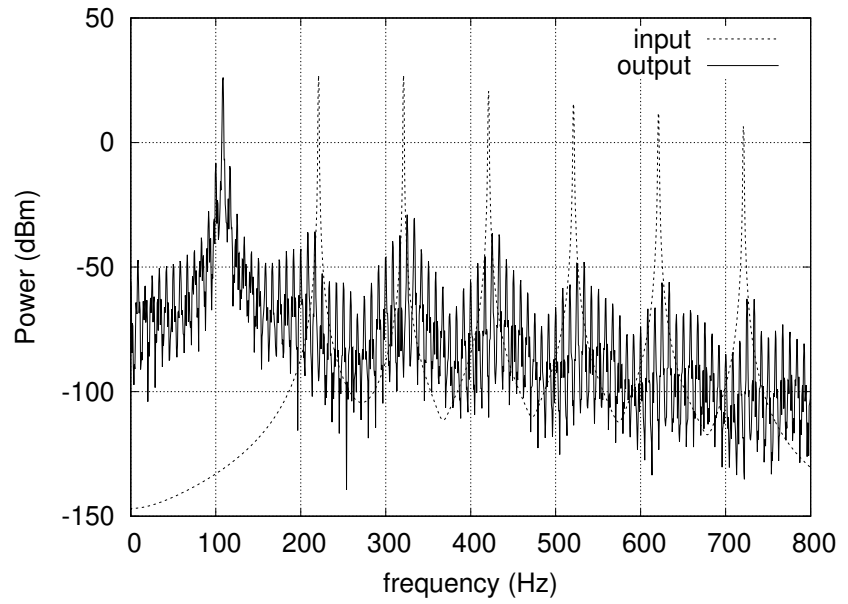

Fig. 15. Example of locking in response to a signal comprised of seven shifted harmonics with amplitude decay as a function of the harmonic number.

precision) to the first mediant of $f_{1} / 2$ and $f_{2} / 3$, i.e., $f_{0}=$ $108 \mathrm{~Hz}$. Another remarkable feature is that, in virtue of the locking mechanism described in Section III, only the residue frequency survives.

Another interesting variation of the input signal, related to the possibility of using the Q-PLL as a model for auditory pitch recognition, is represented by a signal which is a harmonic complex with two-to-several components under pitch shift [7]. This stimulus is the basis of several psychoacoustics experiments (see, e.g., [16], [24]-[27]). Although the argument is very complex and stimuli can be very different, to provide an example: an input signal comprised of the first 7 harmonics of a sine wave of frequency $100 \mathrm{~Hz}$, shifted by the same amount used in previuos pitch-shift examples $(\Delta f=21 \mathrm{hz})$, is fed into the Q-PLL. Figure 15 displays the spectrum of the input and the response signals. The response remains stable to $f_{0}=\left(f_{1}+f_{2}\right) /(2+3)=108.4 \mathrm{~Hz}$ under significant variations in the different magnitudes of the input components, provided 
that the amplitudes decay towards high harmonic numbers (typical of musical sounds).

The resulting spectrum is more complex than that reported in Figure 14, displaying not only the locking peak, but also a number of lower power components. This happens because in this case the low frequency components resulting from the sub-Nyquist sampling (see Section III) do not mutually cancel as in the case of a stimulus comprising only two components of the same amplitude.

\section{CONClusion}

A new nonlinear circuit which can maintain a locked state when forced by two incommensurate frequencies has been presented. The circuit was developed in a biologically inspired paradigm within the framework of dynamical systems theory. Locking states are characterized by the generation of a third frequency which forms a so-called three-frequency resonance together with the forcing frequencies. Moreover, it has been demonstrated that these resonances characterize, in turn, the pitch of complex sounds when the fundamental component is absent. Thanks to this property, the circuit can emulate one of the main psycho-acoustical parameters of auditory perception. Thus, it opens the way for the development of real-time auditory processors which can be utilized in a diversity of applications, from medicine (hearing aids, artificial cochlea), to robotics (artificial senses), to industrial and consumer electronics (by including auditory-like processing in audio applications, as a natural extension of the universal PLL). This new circuit, being orbitally and structurally stable, thanks to a control strategy based on sample-andhold feedback, supersedes former attempts (such as coupled PLLs) and prompts further study regarding optimization of its features and performance in the light of future technological applications.

\section{ACKNOWLEDGEMENTS}

The Italian [10] and International [11] Patent Applications were financially supported by CNR. The authors would like to thank Oreste Piro and Julyan Cartwright for illuminating discussions and Giuliano Gavina for the electronic implementation of an analog version of the circuit.

\section{REFERENCES}

[1] H. de Bellescise, "La réception synchrone," Onde Electrique, vol. 11, pp. 230-240, 1932.

[2] R. Urtel, "Entrainment and synchronization of oscillations," Z. Techn. Phys., vol. 11, pp. 460-465, 1938.

[3] N. Margaris and P. Mastorocostas, "On the nonlinear behavior of the analog phase-locked loop: Synchronization," IEEE Trans. Ind. Electron., vol. 43, no. 6, pp. 621-629, 1997.

[4] W. Mathis and J. Bremen, "Modelling and design concepts for electronic oscillators and its synchronization," The Open Cybernetics \& Systemics Journal, vol. 3, pp. 47-60, 2009.

[5] O. Calvo, J. H. E. Cartwright, D. L. González, O. Piro, and O. Rosso, "Three-frequency resonances in dynamical systems," Int. J. Bifurcation \& Chaos, vol. 9, no. 11, pp. 2181-2187, 1999.

[6] J. H. E. Cartwright, D. L. González, and O. Piro, "Nonlinear dynamics of the perceived pitch of complex sounds," Phys. Rev. Lett., vol. 82, pp. 5389-5392, 1999.

[7] — " "Pitch perception: A dynamical-systems perspective," Proc. Natl Acad. Sci. USA, vol. 98, pp. 4855-4859, 2001.
[8] - "Universality in three-frequency resonances," Phys. Rev. E, vol. 59, pp. 2902-2906, 1999.

[9] O. Calvo, J. H. E. Cartwright, D. L. González, O. Piro, and F. Sportolari, "Three-frequency resonances in coupled phase-locked loops," IEEE Trans. Circuits Syst., vol. 47, pp. 491-497, 2000.

[10] D. L. González and A. Maurizi, "Circuit and method for modeling and emulating the pitch perception of complex sounds," Italian patent application IT102 019000021111 , Nov. 13, 2019.

[11] - "Circuit and method for modeling and emulating the pitch perception of complex sounds," International patent application PCT/EP2020/081 140, Nov. 5, 2020.

[12] C. Huygens, Euvres Complètes de Christiaan Huygens. Societé Hollandaise des Sciences, 1888-1950, vol. 17, p. 185.

[13] B. Escribano, J. Vanyo, I. Tuval, J. H. E. Cartwright, D. L. González, O. Piro, and T. Tel, "Dynamics of tidal synchronization and orbit circularization of celestial bodies," Phys. Rev. E, vol. 78, p. 036216, 2008.

[14] B. Escribano, J. Vanyo, I. Tuval, J. H. Cartwright, D. L. Gonzalez, O. Piro, and T. Tel, "A minimal dynamical model for tidal synchronization and orbit circularization," Celestial Mechanics and Dynamical Astronomy, vol. 209, pp. 181-200, 2010.

[15] T. Kapitaniak and J. Wojewoda, Attractors of Quasiperiodically Forced Systems. World Scientific, 1994.

[16] J. F. Schouten, R. J. Ritsma, and B. L. Cardozo, "Pitch of the residue," J. Acoust. Soc. Am., vol. 34, pp. 1418-1424, 1962.

[17] H. Helmholtz, Die Lehre von den Tonempfindungen als physiologische Grundlage für die Theorie der Musik. Vieweg, Braunschweig, 1863.

[18] F. L. Wightman, "The pattern transformation model of pitch," J. Acoust. Soc. Am., vol. 54, pp. 407-416, 1973.

[19] E. Terhardt, "Pitch, consonance, and harmony," J. Acoust. Soc. Am., vol. 55, pp. 1061-1069, 1974.

[20] A. de Chevigné, "Pitch perception models," in Pitch - Neural Coding and Perception, ser. Springer handbook of auditory research, C. J. Plack, A. J. Oxenham, R. R. Fay, and A. N. Popper, Eds. Springer ScienceBusiness Media, Inc., 2005, vol. 24, ch. 6.

[21] G. H. Hardy and E. M. Wright, An introduction to the theory of numbers. Oxford: Clarendon Press, 1938.

[22] J. H. E. Cartwright, J. Douthett, D. L. González, R. Krantz, and O. Piro, "Two musical paths to the Farey series and devil's staircase," J. Math. Music, vol. 4, no. 1, pp. 57-74, 2010.

[23] W. M. Hartmann, Signals, Sound, and Sensation, ser. Modern Acoustics and Signal Processing. Springer, 1998.

[24] R. J. Ritsma and F. L. Engel, "Pitch of frequency-modulated signals," J. Acoust. Soc. Am., vol. i36, no. 9, pp. 1637-1644, 1964.

[25] G. F. Smorenburg, "Pitch perception of two-frequency stimuli," J. Acoust. Soc. Am., vol. 48, no. 4, pp. 924-942, 1970.

[26] R. D. Patterson, "The effect of relative phase and the number of components on residue pitch," J. Acoust. Soc. Am., vol. 53, no. 6, pp. $1565-1572,1973$.

[27] M. Greenhough and W. R. P., "Experiments on the pitch of signals derived from three-tone complexes," Journal of Sound and Vibration, vol. 41 , no. 4 , pp. $451-461,1975$. 


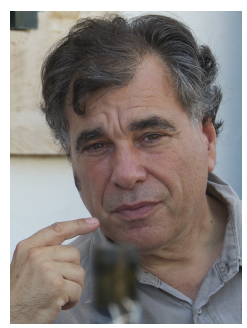

Diego Luis González Diego L. González was born in Buenos Aires, Argentina, in 1951. He qualified as Professor of pianoforte in 1971. He received his degree in physics from the University of $\mathrm{La}$ Plata in Argentina in 1981 and $\mathrm{PhD}$ in Theoretical Physics from the same University in 1987. His $\mathrm{PhD}$ focused on the study of synchronisation and chaos in nonlinear oscillators. He worked from 1988 in the CNR LAMEL Institute (now IMM) in the field of microelectronics and from 1999 to 2008 he collaborated with the Acoustical Laboratory of the St. George School Foundation and the CNR in Venice. At present he is an Associate Researcher at the CNR Institute for Microsystems and Microelectronics (IMM), also working at the Statistical Sciences Department of Bologna University. His main research interests are the theory of lowdimensional nonlinear systems and its application to modelling complex dynamical systems and, in particular, biological ones. In this latter field, he has formulated and developed an original theory of the pitch perception of complex sounds based on multi-frequency resonances and a mathematical model of the genetic code with consequences on its putative origin and evolution.

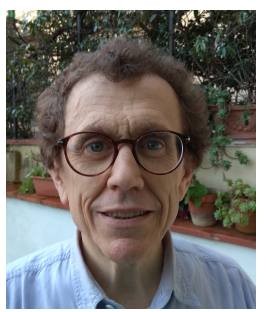

Lorenzo Grassi Studied at the University of Florence, Italy, where he earned his Laurea (Italian equivalent MSc) in Physics in 1994. 1995-97 EEC HCM Fellowship at the Max Planck Institut für Meteorologie (MPIfM), Hamburg, Germany. Spent the next four years at the Institute of Atmospheric Sciences and Climate (ISAC) of Italy's National Research Council (CNR). His research focused on optical atmospheric remote sensing and, in particular on LIDAR (LIght Detecting And Ranging), DIAL (DIfferential Absorption Lidar), DOAS (Differential Optical Absorption Spectroscopy) and the optical design of apparatus for in situ air quality monitoring. Worked as a consultant in optics and in software development for industrial automation. Since 2004, shareholder and project manager of Asper S.r.l., in charge of industrial and research projects, and since 2005 he is the sole director of the company.

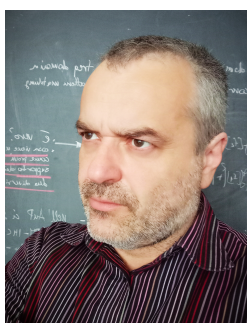

Alberto Maurizi Alberto Maurizi obtained his Laurea (Italian equivalent of $\mathrm{MSc}$ ), cum laude, in physics from the University of Bologna, Italy, in 1992. His education continued with the support of the Italian National Research Council (CNR) at the Institute of Atmospheric Sciences and Climate (CNR-ISAC) and subsequently supported by PostDoc EC grants (EC HCM Programme). Promoted to a permanent position in 2001 at CNR-ISAC, he worked on complex geophysical problems (mainly turbulence in geophysical flows). More recently, he moved to the CNR-IMM (Institute of Microelectronics and Microsystems) to follow his growing interest in dynamical systems and their application to sound perception modelling. 